SSU-HEP-12/08

\title{
Hyperfine structure of excited state of muonic helium atom
}

\author{
A.A. Krutov* \\ Samara State University, Pavlov street 1, 443011, Samara, Russia \\ A.P. Martynenkd† \\ Samara State University, Pavlov Street 1, 443011, Samara, Russia and \\ Samara State Aerospace University named after S.P. Korolyov, \\ Moskovskoye Shosse 34, 443086, Samara, Russia
}

\begin{abstract}
The recoil, vacuum polarization and electron vertex corrections of first and second orders in the fine structure constant $\alpha$ and the ratio of electron to muon and electron to $\alpha$-particle masses are calculated in the hyperfine splitting of the $1 s_{1 / 2}^{(e)} 2 s_{1 / 2}^{(\mu)}$ state of muonic helium atom $\left(\mu e_{2}^{4} \mathrm{He}\right)$ on the basis of a perturbation theory. We obtain total result for the muonically excited state hyperfine splitting $\Delta \nu^{h f s}=4295.66 \mathrm{MHz}$ which improves previous calculations due to the account of new corrections and more accurate treatment of the electron vertex contribution.
\end{abstract}

PACS numbers: 31.30.Jv, 12.20.Ds, 32.10.Fn

\section{INTRODUCTION}

The muonic helium atom $\left(\mu e_{2}^{4} H e\right)$ represents the simple three-body atomic system. The interaction between magnetic moments of the muon and electron leads to the hyperfine structure (HFS) of the $S$-wave energy levels. Whereas the ground state hyperfine splitting was measured many years ago with sufficiently high accuracy in [1, 2] $\Delta \nu_{e x p}^{h f s}=4465.004(29) \mathrm{MHz}$, the excited states in muonic helium atom were studied significantly smaller. Nevertheless, during the formation of muonic helium atoms the negative muon is kept by positive $\alpha$ particle and is found to be in excited state. The lifetime of excited state with the muon locating in the $2 S$-state is sufficient to measure its hyperfine splitting. This can be important for the check of quantum electrodynamics for three-particle muonic bound states.

At present, hydrogen-like bound-state physics in QED is reliably developed [3 7]. Contrary to two-particle bound states whose energy levels were accurately calculated in quantum electrodynamics [3-7], the hyperfine splitting of three-particle muonic states was studied on the basis of perturbation theory (PT) and variational approach with smaller accuracy 8 12]. There are several calculations devoted to HFS of the excited state $1 s_{1 / 2}^{(e)} 2 s_{1 / 2}^{(\mu)}$ in muonic helium atom [13 16]. In these papers different order corrections to HFS were studied by

*Electronic address: aakrutov@samsu.ru

${ }^{\dagger}$ Electronic address: a.p.martynenko@samsu.ru 
perturbation theory, variational method and Born-Oppenheimer approach. The accuracy of the calculations lies in the interval of several tenth parts of MHz. The variational method gives high numerical accuracy of the calculation what was demonstrated in [10, 12, 13, 15]. The correlated wave functions of the state $1 s_{1 / 2}^{(e)} 2 s_{1 / 2}^{(\mu)}$ were constructed and higher order corrections to the hyperfine splitting were also accounted for in this approach in [15]. It is appropriate to mention here that three-particle kinematics was chosen for the description of muonic helium in [8, 9, 13 15] by different ways. So, numerical results of obtained corrections of different order in $\alpha$ and the ratio of the particle masses are difficult for direct comparison.

Bound particles in muonic helium have different masses $m_{e} \ll m_{\mu} \ll m_{\alpha}$. As a result the muon and $\alpha$-particle compose the pseudonucleus $\left(\mu_{2}^{4} \mathrm{He}\right)^{+}$and muonic helium atom looks as a two particle system in first approximation. The existence of such mass hierarchy enables to formulate a perturbation theory for the calculation of the energy levels. The perturbation theory approach to the investigation of the hyperfine structure of muonic helium based on nonrelativistic Schrödinger equation was developed in [8]. Using their method we describe the excited state in three-particle bound system $\left(\mu e_{2}^{4} H e\right)$ by the Hamiltonian:

$$
\begin{gathered}
H=H_{0}+\Delta H+\Delta H_{r e c}, \quad H_{0}=-\frac{1}{2 M_{\mu}} \nabla_{\mu}^{2}-\frac{1}{2 M_{e}} \nabla_{e}^{2}-\frac{2 \alpha}{x_{\mu}}-\frac{\alpha}{x_{e}}, \\
\Delta H=\frac{\alpha}{x_{\mu e}}-\frac{\alpha}{x_{e}}, \quad \Delta H_{r e c}=-\frac{1}{m_{\alpha}} \nabla_{\mu} \cdot \nabla_{e},
\end{gathered}
$$

where $\mathbf{x}_{\mu}$ and $\mathbf{x}_{\mathbf{e}}$ are the coordinates of the muon and electron relative to the helium nucleus, $M_{e}=m_{e} m_{\alpha} /\left(m_{e}+m_{\alpha}\right), M_{\mu}=m_{\mu} m_{\alpha} /\left(m_{\mu}+m_{\alpha}\right)$ are the reduced masses of subsystems $\left(e_{2}^{4} \mathrm{He}\right)^{+}$and $\left(\mu_{2}^{4} \mathrm{He}\right)^{+}$. The hyperfine part of the Hamiltonian contributing to HFS of $S$ states is

$$
\Delta H^{h f s}=-\frac{8 \pi \alpha\left(1+\kappa_{\mu}\right)}{3 m_{e} m_{\mu}} \frac{\left(\boldsymbol{\sigma}_{e} \boldsymbol{\sigma}_{\mu}\right)}{4} \delta\left(\mathbf{x}_{\mu}-\mathbf{x}_{\mathbf{e}}\right)
$$

where $\boldsymbol{\sigma}_{e}$ and $\boldsymbol{\sigma}_{\mu}$ are the spin matrices of the electron and muon. In initial approximation the wave function of the excited state $1 s_{1 / 2}^{(e)} 2 s_{1 / 2}^{(\mu)}$ has the form:

$$
\Psi_{0}\left(\mathbf{x}_{\mathbf{e}}, \mathbf{x}_{\mu}\right)=\psi_{e}\left(\mathbf{x}_{\mathbf{e}}\right) \psi_{\mu}\left(\mathbf{x}_{\mu}\right)=\frac{1}{2 \sqrt{2} \pi}\left(W_{\mu} W_{e}\right)^{3 / 2}\left(1-\frac{W_{\mu} x_{\mu}}{2}\right) e^{-W_{\mu} x_{\mu}} e^{-W_{e} x_{e}},
$$

where $W_{\mu}=2 \alpha M_{\mu}, W_{e}=\alpha M_{e}$. Then basic contribution to the singlet-triplet hyperfine splitting can be calculated analytically from contact interaction (3):

$$
\Delta \nu_{0}^{h f s}=<\frac{8 \pi \alpha\left(1+\kappa_{\mu}\right)}{3 m_{e} m_{\mu}} \delta\left(\mathbf{x}_{\mu}-\mathbf{x}_{\mathbf{e}}\right)>=\frac{\nu_{F}\left(1+\kappa_{\mu}\right)}{\left(1+\frac{M_{e}}{M_{\mu}}\right)^{5}}\left(1-\frac{M_{e}}{M_{\mu}}+\frac{M_{e}^{2}}{M_{\mu}^{2}}\right), \quad \nu_{F}=\frac{8 \alpha^{4} M_{e}^{3}}{3 m_{e} m_{\mu}} .
$$

Numerically, the Fermi splitting is equal $\nu_{F}=4516.915 \mathrm{MHz}$. We express further the hyperfine splitting contributions in the frequency unit using the relation $\Delta E^{h f s}=2 \pi \hbar \Delta \nu^{h f s}$. For the sake of definiteness we write all numerical values with an accuracy $0.001 \mathrm{MHz}$. The recoil correction of first order in the ratio $M_{e} / M_{\mu}$ in (5) amounts to $\Delta \nu_{r e c}^{h f s}=$ $-6 \frac{M_{e}}{M_{\mu}} \nu_{F}=-134.769 \mathrm{MHz}$. The muon anomalous magnetic moment correction is equal to $\nu_{F} \kappa_{\mu}=5.266 \mathrm{MHz}$. Modern numerical values of fundamental physical constants are taken 
from the paper [17]: the electron mass $m_{e}=0.510998928(11) \cdot 10^{-3} \mathrm{GeV}$, the muon mass $m_{\mu}=0.1056583715(35) \mathrm{GeV}$, the fine structure constant $\alpha^{-1}=137.035999074(44)$, the helium mass $m_{\alpha}=3.727379240(82) \mathrm{GeV}$, the electron anomalous magnetic moment $\kappa_{e}=$ $1.15965218076(27) \cdot 10^{-3}$, the muon anomalous magnetic moment $\kappa_{\mu}=1.16592091(63) \cdot 10^{-3}$.

In this work we aim to extend the approach of Lakdawala and Mohr [8] to the case of HFS of the excited state $1 s_{1 / 2}^{(e)} 2 s_{1 / 2}^{(\mu)}$ in muonic helium atom. The terms of the Hamiltonian $\Delta H$ and $\Delta H_{\text {rec }}$ give important recoil corrections in second order perturbation theory. A feature that distinguishes light muonic atoms among other simplest atoms is that the structure of their energy levels depends strongly on the vacuum polarization (VP) effects, nuclear structure and recoil corrections [18 21]. So, we investigate the role of all such potentially important corrections to excited state HFS in muonic helium. Another purpose of our study consists in the improved evaluation of the electron one-loop vertex corrections to HFS of order $\alpha^{5}$ using analytical expression of the electron electromagnetic form factors [22].

\section{RECOIL CORRECTIONS}

Along with small parameter $\alpha$ there are two other small parameters in this task equal to the ratio of the particle masses $M_{e} / M_{\mu}$ and $M_{e} / m_{\alpha}$. They determine the recoil corrections which can be calculated by the perturbation theory. First recoil correction is presented in (5). Let us consider the recoil contribution in second order perturbation theory. For its study we use the initial expression for second order correction to the HFS from [8, 22]:

$$
\begin{gathered}
\Delta \nu^{h f s,(2)}=\frac{16 \pi \alpha\left(1+\kappa_{\mu}\right)}{3 m_{e} m_{\mu}} \int d \mathbf{x}_{1} \int d \mathbf{x}_{2} \int d \mathbf{x}_{3} \psi_{\mu}^{*}\left(\mathbf{x}_{3}\right) \psi_{e 0}^{*}\left(\mathbf{x}_{3}\right) \times \\
\sum_{n, m}^{\prime} \frac{\psi_{\mu, n}\left(\mathbf{x}_{3}\right) \psi_{e, m}\left(\mathbf{x}_{3}\right) \psi_{\mu, n}^{*}\left(\mathbf{x}_{2}\right) \psi_{e, m}^{*}\left(\mathbf{x}_{1}\right)}{E_{\mu}+E_{e}-E_{\mu, n}-E_{e, m}} \Delta H\left(\mathbf{x}_{2}, \mathbf{x}_{1}\right) \psi_{\mu}\left(\mathbf{x}_{2}\right) \psi_{e}\left(\mathbf{x}_{1}\right),
\end{gathered}
$$

where the superscript (2) designates the correction in second order perturbation theory. The prime near the sum symbol means that the term with $n=2, m=0$ is excluded. $\Delta H\left(\mathbf{x}_{1}, \mathbf{x}_{2}\right)$ is determined by (2), $E_{\mu}$ is the Coulomb muon energy in the $2 S$-state, $E_{e}$ is the Coulomb electron energy in the $1 S$-state. It is useful to extract in (6) several contributions. If the muon is in the $2 S$ intermediate state $\left(E_{\mu}-E_{\mu, n=2}=0\right)$ then

$$
\Delta \nu_{2 S}^{h f s,(2)}=\frac{16 \pi \alpha\left(1+\kappa_{\mu}\right)}{3 m_{e} m_{\mu}} \int d \mathbf{x}_{1} \int d \mathbf{x}_{3}\left|\psi_{\mu}\left(\mathbf{x}_{3}\right)\right|^{2} \psi_{e}\left(\mathbf{x}_{3}\right) \Delta V_{\mu}\left(\mathbf{x}_{1}\right) \psi_{e}\left(\mathbf{x}_{1}\right) G_{e}\left(\mathbf{x}_{1}, \mathbf{x}_{3}\right),
$$

where the Coulomb reduced Green's function of the electron (see [23])

$$
\begin{gathered}
G_{e}\left(\mathbf{x}_{1}, \mathbf{x}_{3}\right)=\sum_{n \neq 0}^{\infty} \frac{\psi_{e, n}\left(\mathbf{x}_{3}\right) \psi_{e, n}^{*}\left(\mathbf{x}_{1}\right)}{E_{e}-E_{e, n}}=-\frac{\alpha M_{e}^{2}}{\pi} e^{-W_{e}\left(x_{1}+x_{3}\right)}\left[\frac{1}{2 W_{e} x_{>}}-\right. \\
\left.-\ln \left(2 W_{e} x_{>}\right)-\ln \left(2 W_{e} x_{<}\right)+E i\left(2 W_{e} x_{<}\right)+\frac{7}{2}-2 C-W_{e}\left(x_{1}+x_{3}\right)+\frac{1-e^{2 W_{e} x_{<}}}{2 W_{e} x_{<}}\right],
\end{gathered}
$$


$x_{<}=\min \left(x_{1}, x_{3}\right), x_{>}=\max \left(x_{1}, x_{3}\right), C=0.577216 \ldots$ is the Euler's constant and $\operatorname{Ei}(x)$ is the exponential-integral function,

$\Delta V_{\mu}\left(\mathbf{x}_{1}\right)=\int d \mathbf{x}_{2} \psi_{\mu}^{*}\left(\mathbf{x}_{2}\right) \Delta H\left(\mathbf{x}_{1}, \mathbf{x}_{2}\right) \psi_{\mu}\left(\mathbf{x}_{2}\right)=-\frac{\alpha}{x_{1}} e^{-W_{\mu} x_{1}}\left[1+\frac{3}{4} W_{\mu} x_{1}+\frac{1}{4} W_{\mu}^{2} x_{1}^{2}+\frac{1}{8} W_{\mu}^{3} x_{1}^{3}\right]$.

Substituting (9) and (8) in (6) we perform analytical integration over all coordinates and expand exact result in the ratio $M_{e} / M_{\mu}$ preserving the terms of third order:

$$
\begin{gathered}
\Delta \nu_{2 S}^{h f s,(2)}=\nu_{F}\left(1+\kappa_{\mu}\right) \frac{M_{e}}{M_{\mu}}\left[\frac{631}{256}+\frac{M_{e}}{M_{\mu}}\left(-14 \ln \frac{M_{e}}{M_{\mu}}-\frac{5291}{512}-14 \ln 2\right)+\right. \\
\left.+\left(\frac{M_{e}}{M_{\mu}}\right)^{2}\left(139 \ln \frac{M_{e}}{M_{\mu}}-\frac{17361}{512}+139 \ln 2\right)\right]=61.127 \mathrm{MHz} .
\end{gathered}
$$

Another correction in (6) is determined by the muon in the $2 P$ intermediate state:

$$
\begin{gathered}
\Delta \nu_{2 P}^{h f s,(2)}=-\frac{16 \pi \alpha^{2} M_{e}^{2}\left(1+\kappa_{\mu}\right) W_{\mu}^{5}}{9 m_{e} m_{\mu}} \int x_{2} d \mathbf{x}_{2} \int x_{3} d \mathbf{x}_{3} \psi_{\mu}^{*}\left(\mathbf{x}_{3}\right) \psi_{e}^{*}\left(\mathbf{x}_{3}\right) \sum_{m=-1}^{1} Y_{1 m}\left(\mathbf{n}_{3}\right) Y_{1 m}^{*}\left(\mathbf{n}_{2}\right) \times \\
\int d \mathbf{x}_{1} e^{-W_{\mu} x_{2} / 2} e^{-W_{\mu} x_{3} / 2} \Delta H\left(\mathbf{x}_{2}, \mathbf{x}_{1}\right) \psi_{\mu}\left(\mathbf{x}_{2}\right) \psi_{e}\left(\mathbf{x}_{1}\right) \sum_{m^{\prime}=-1}^{1} Y_{1 m^{\prime}}\left(\mathbf{n}_{1}\right) Y_{1 m^{\prime}}^{*}\left(\mathbf{n}_{3}\right) g_{11}\left(W_{e} x_{1}, W_{e} x_{3}\right),
\end{gathered}
$$

where the unit vectors $\mathbf{n}_{i}=\mathbf{r}_{i} / r(i=1,2,3)$, the partial Green's function (see [24] $)$

$g_{11}\left(W_{e} x_{1}, W_{e} x_{3}\right)=e^{-W_{e}\left(x_{1}+x_{3}\right)}\left(\frac{1}{4 W_{e}^{2} x_{>}^{2}}+\frac{1}{2 W_{e} x_{>}}+\frac{1}{2}\right)\left(\frac{e^{2 W_{e} x_{<}}}{4 W_{e}^{2} x_{<}^{2}}-\frac{1}{4 W_{e}^{2} x_{<}^{2}}-\frac{1}{2 W_{e} x_{<}}-\frac{1}{2}\right)$.

Integrating analytically in (11), we can present the expansion of final result in $M_{e} / M_{\mu}$ up to terms of third order in the form:

$$
\Delta \nu_{2 P}^{h f s,(2)}=\nu_{F}\left(1+\kappa_{\mu}\right)\left(-\frac{837}{256} \frac{M_{e}}{M_{\mu}}+\frac{12141}{512} \frac{M_{e}^{2}}{M_{\mu}^{2}}-\frac{43335}{512} \frac{M_{e}^{3}}{M_{\mu}^{3}}\right)=-70.919 \mathrm{MHz} .
$$

In addition, there exists in (6) the contribution corresponding to excited states of the muon:

$$
\begin{gathered}
\Delta \nu_{n \neq 2 S, 2 P}^{h f s,(2)}=-\frac{16 \pi \alpha\left(1+\kappa_{\mu}\right)}{3 m_{e} m_{\mu}} \int d \mathbf{x}_{1} \int d \mathbf{x}_{2} \int d \mathbf{x}_{3} \psi_{\mu}^{*}\left(\mathbf{x}_{3}\right) \psi_{e}^{*}\left(\mathbf{x}_{3}\right) \times \\
\sum_{n \neq 2} \psi_{\mu, n}\left(\mathbf{x}_{3}\right) \psi_{\mu, n}^{*}\left(\mathbf{x}_{2}\right) G_{e}\left(\mathbf{x}_{3}, \mathbf{x}_{1}, E_{\mu}+E_{e}-E_{\mu, n}\right) \frac{\alpha}{\left|\mathbf{x}_{2}-\mathbf{x}_{1}\right|} \psi_{\mu}\left(\mathbf{x}_{2}\right) \psi_{e}\left(\mathbf{x}_{1}\right) .
\end{gathered}
$$

It can be calculated analytically in the leading order in $M_{e} / M_{\mu}$ if we replace the exact Green's function of the electron $G_{e}\left(\mathbf{x}_{3}, \mathbf{x}_{1}, E_{\mu}+E_{e}-E_{\mu, n}\right)$ by free Green's function

$$
G_{e, 0}\left(\mathbf{x}_{3}, \mathbf{x}_{1}, E_{\mu}+E_{e}-E_{\mu, n}\right)=\frac{M_{e}}{2 \pi} \frac{e^{-b\left|\mathbf{x}_{3}-\mathbf{x}_{1}\right|}}{\left|\mathbf{x}_{3}-\mathbf{x}_{1}\right|}, \quad b=\sqrt{2 M_{e}\left(E_{\mu, n}-E_{\mu}-E_{e}\right)} .
$$


Replacing also the electron wave function by its value at the origin $\psi_{e}(0)$ and integrating over the coordinate $\mathbf{x}_{1}$ in (14) we obtain (see [8, 22]):

$$
I_{1}=\int d \mathbf{x}_{1} \frac{e^{-b\left|\mathbf{x}_{3}-\mathbf{x}_{1}\right|}}{\left|\mathbf{x}_{3}-\mathbf{x}_{1}\right|\left|\mathbf{x}_{2}-\mathbf{x}_{1}\right|}=4 \pi\left[\frac{1}{b}-\frac{1}{2}\left|\mathbf{x}_{2}-\mathbf{x}_{3}\right|+\frac{b}{6}\left|\mathbf{x}_{2}-\mathbf{x}_{3}\right|^{2}+\ldots\right],
$$

where the expansion in $b / W_{\mu} \sim \sqrt{M_{e} / M_{\mu}}$ is performed. The term $1 / b$ does not contribute. Taking second term in (16), we use further the completeness condition:

$$
\sum_{n \neq 2 P, 2 S} \psi_{\mu, n}\left(\mathbf{x}_{3}\right) \psi_{\mu, n}^{*}\left(\mathbf{x}_{2}\right)=\delta\left(\mathbf{x}_{3}-\mathbf{x}_{2}\right)-\psi_{\mu, 2 S}\left(\mathbf{x}_{3}\right) \psi_{\mu, 2 S}^{*}\left(\mathbf{x}_{2}\right)-\sum_{m=-1}^{1} \psi_{\mu, 2 P, m}\left(\mathbf{x}_{3}\right) \psi_{\mu, 2 P, m}^{*}\left(\mathbf{x}_{2}\right)
$$

where we write explicitely subscripts $2 S$ and $2 P$ corresponding to $n=2$ muonic states. Then the contribution of each component from (17) can be calcuated analytically together with the term $\frac{1}{2}\left|\mathbf{x}_{2}-\mathbf{x}_{3}\right|$. We obtain following results corresponding to the $2 S$ and $2 P$ states in (17):

$$
\Delta \nu_{1}^{h f s,(2)}=-\nu_{F}\left(1+\kappa_{\mu}\right) \frac{M_{e}}{M_{\mu}} \frac{2167}{256}, \quad \Delta \nu_{2}^{h f s,(2)}=\nu_{F}\left(1+\kappa_{\mu}\right) \frac{M_{e}}{M_{\mu}} \frac{837}{256} .
$$

Note that the order $O\left(M_{e} / M_{\mu}\right)$ corrections in (13) and (18) corresponding to $2 P$-state cancel in the sum. To increase the accuracy of the calculation we analyze third term in (16). It can be expressed in terms of the following sum [3, 8]:

$$
S_{1 / 2}=\sum_{n \neq 2}\left(\frac{E_{\mu, n}-E_{\mu, 2}}{R_{\mu}}\right)^{1 / 2}|<\mu, 2 S| \frac{\mathbf{x}}{a_{\mu}}|\mu, n>|^{2} .
$$

The discrete and continuum states contributions to (19) are equal correspondingly [25]:

$$
\begin{gathered}
S_{1 / 2}^{d}=\sum_{n>2} \frac{2^{16} n^{6}\left(n^{2}-1\right)(n-2)^{2 n-6+\frac{1}{2}}}{(n+2)^{2 n+6-\frac{1}{2}}}=4.918 \ldots, \\
S_{1 / 2}^{c}=\int_{0}^{\infty} 2^{5}\left(k^{2}+\frac{1}{4}\right)^{1 / 2} \frac{\left(k^{2}+1\right)}{\left(k^{2}+\frac{1}{4}\right)^{6}} \frac{k}{1-e^{-\frac{2 \pi}{k}}} e^{-\frac{4}{k} \operatorname{arctg}(2 k)}=1.563 \ldots
\end{gathered}
$$

As a result the contribution of third term from the expansion (16) is equal to

$$
\Delta \nu_{3}^{h f s,(2)}=\nu_{F}\left(1+\kappa_{\mu}\right) \frac{2}{3}\left(\frac{M_{e}}{M_{\mu}}\right)^{3 / 2} S_{1 / 2}=6.853 M H z .
$$

Another set of recoil corrections in second order PT is determined by the perturbation $\Delta H_{r e c}=-\frac{1}{m_{\alpha}} \nabla_{\mu} \nabla_{e}$. In this case the basic contribution to HFS is the following

$$
\begin{gathered}
\Delta \nu_{\alpha}^{h f s}=-\frac{32 \pi \alpha^{3} M_{e} M_{\mu}}{3 m_{\alpha} m_{e} m_{\mu}} \int d \mathbf{x}_{1} \int d \mathbf{x}_{2} \int d \mathbf{x}_{3} \psi_{\mu}^{*}\left(\mathbf{x}_{3}\right) \psi_{e}^{*}\left(\mathbf{x}_{3}\right) \times \\
\sum_{n, m} \frac{\psi_{\mu, n}\left(\mathbf{x}_{3}\right) \psi_{e, m}\left(\mathbf{x}_{3}\right) \psi_{\mu, n}^{*}\left(\mathbf{x}_{2}\right) \psi_{e, m}^{*}\left(\mathbf{x}_{1}\right)}{E_{\mu}+E_{e}-E_{\mu, n}-E_{e, m}} \frac{W_{e}^{3 / 2} W_{\mu}^{3 / 2}}{2 \pi \sqrt{2}} e^{-W_{e} x_{1}} e^{-W_{\mu} x_{2} / 2}\left(2-\frac{W_{\mu} x_{2}}{2}\right)\left(\mathbf{n}_{1} \mathbf{n}_{2}\right),
\end{gathered}
$$


where we substitute

$$
\nabla_{2} \nabla_{1} \psi_{\mu}\left(\mathbf{x}_{2}\right) \psi_{e}\left(\mathbf{x}_{1}\right)=\frac{\alpha^{2} M_{e} M_{\mu} W_{e}^{3 / 2} W_{\mu}^{3 / 2}}{2 \pi \sqrt{2}} e^{-W_{e} x_{1}} e^{-W_{\mu} x_{2} / 2}\left(2-\frac{W_{\mu} x_{2}}{2}\right)\left(\mathbf{n}_{1} \mathbf{n}_{2}\right) .
$$

The muon $2 S$-state does not contribute in (22). In the case of the $2 P$ muon intermediate state when $\psi_{\mu, n}=\psi_{\mu, 2 P}$, we can integrate at first over angle variables. Then we obtain the radial integral $\int_{0}^{\infty} x_{2}^{3} d x_{2} e^{-x_{2}}\left(2-x_{2} / 2\right)=0$. So, we should consider the muon intermediate states with $n \neq 2$ in (22). Acting as in (14) we obtain:

$$
\begin{aligned}
\Delta \nu_{\alpha}^{h f s,(1)}(n \neq 2)= & \frac{2 \alpha^{3} M_{e}^{2} M_{\mu} W_{e}^{3} W_{\mu}^{3}}{3 m_{\alpha} m_{e} m_{\mu} \pi^{2}} \int d \mathbf{x}_{1} \int d \mathbf{x}_{2} \int d \mathbf{x}_{3}\left(1-\frac{W_{\mu} x_{3}}{2}\right) e^{-W_{\mu} x_{3} / 2} e^{-W_{\mu} x_{2} / 2} \times \\
& \left(2-\frac{W_{\mu} x_{2}}{2}\right)\left(\mathbf{n}_{1} \mathbf{n}_{2}\right) \sum_{n \neq 2} \psi_{\mu, n}\left(\mathbf{x}_{3}\right) \psi_{\mu, n}^{*}\left(\mathbf{x}_{2}\right) \frac{e^{-b\left|\mathbf{x}_{3}-\mathbf{x}_{1}\right|}}{\left|\mathbf{x}_{3}-\mathbf{x}_{1}\right|}
\end{aligned}
$$

Then we can integrate over $\mathbf{x}_{1}$ and expand the result over small parameter $b$ :

$$
J_{\alpha}=\int d \mathbf{x}_{1}\left(\mathbf{n}_{1} \mathbf{n}_{2}\right) \frac{e^{-b\left|\mathbf{x}_{3}-\mathbf{x}_{1}\right|}}{\left|\mathbf{x}_{3}-\mathbf{x}_{1}\right|}=2 \pi\left(\mathbf{n}_{2} \mathbf{n}_{3}\right)\left[\frac{4 x_{3}}{3 b}-\frac{x_{3}^{2}}{2}+\frac{2 b x_{3}^{3}}{15}+\ldots\right] .
$$

Taking first term in square brackets we obtain two radial integrals which determine the contribution (24) for the transition $2 S \rightarrow n P(n>2)$ :

$$
J_{1}=\int_{0}^{\infty} e^{-x / 2} e^{-x / n} \frac{2 x}{n} L_{n-2}^{3}\left(\frac{2 x}{n}\right) x^{2} d x, \quad J_{2}=\int_{0}^{\infty} R_{2 S}(x) R_{n 1}(x) x^{3} d x .
$$

Extracting two contributions to the product of $J_{1}$ and $J_{2}$ corresponding to descrete and continuum states we present the correction (24) in the form [25]:

$$
\begin{gathered}
\Delta \nu_{\alpha}^{h f s,(1)}(n \neq 2)=\nu_{F}\left(1+\kappa_{\mu}\right) \frac{4 M_{e}}{3 m_{\alpha}} \sqrt{\frac{M_{e}}{M_{\mu}}} S_{j_{1} j_{2}}=0.189 \mathrm{MHz}, \\
S_{j_{1} j_{2}}=\sum_{n>2} \frac{2^{15}(n-2)^{2 n-5-\frac{1}{2}} n^{6}\left(n^{2}-1\right)}{(n+2)^{2 n+5+\frac{1}{2}}}+\int_{0}^{\infty} \frac{2^{15} k\left(k^{2}+1\right) d k}{\left(4 k^{2}+1\right)^{11 / 2}\left(1-e^{-\frac{2 \pi}{k}}\right)} e^{-\frac{4}{k} \arctan (2 k)}=3.24101 \ldots
\end{gathered}
$$

Second term $\left(-\pi\left(\mathbf{n}_{2} \mathbf{n}_{3}\right) x_{3}^{2}\right)$ in the expansion (25) is not dependent on $n$. So, we can present its contribution using the completeness condition:

$$
\begin{gathered}
\Delta \nu_{\alpha}^{h f s,(2)}(n \neq 2)=\frac{4 \alpha^{3} M_{e}^{2} M_{\mu} W_{e}^{3} W_{\mu}^{3}}{3 \pi m_{\alpha} m_{e} m_{\mu}} \int d \mathbf{x}_{2} \int x_{3}^{2} d \mathbf{x}_{3}\left(1-\frac{W_{\mu} x_{3}}{2}\right) e^{-W_{\mu} x_{3} / 2} e^{-W_{\mu} x_{2} / 2} \times \\
\left(2-\frac{W_{\mu} x_{2}}{2}\right)\left(\mathbf{n}_{2} \mathbf{n}_{3}\right)\left[\delta\left(\mathbf{x}_{2}-\mathbf{x}_{3}\right)-\psi_{\mu, 2 S}\left(\mathbf{x}_{3}\right) \psi_{\mu, 2 S}^{*}\left(\mathbf{x}_{2}\right)-\sum_{m=-1}^{1} \psi_{\mu, 2 P, m}\left(\mathbf{x}_{3}\right) \psi_{\mu, 2 P, m}^{*}\left(\mathbf{x}_{2}\right)\right] .
\end{gathered}
$$

Only the $\delta$-term in (28) gives nonzero correction of second order in the ratio of particle masses:

$$
\Delta \nu_{\alpha}^{h f s,(2)}(n \neq 2)=\nu_{F} \frac{24 M_{e}^{2}}{m_{\alpha} M_{\mu}}=0.074 M H z .
$$




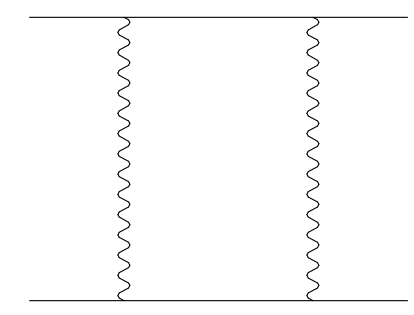

a

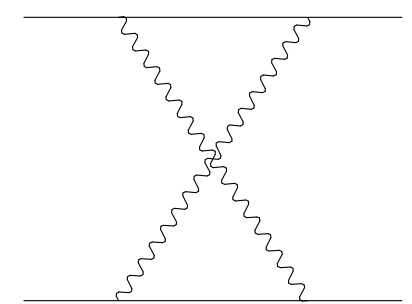

$\mathrm{b}$

FIG. 1: Two photon exchange amplitudes in the electron-muon hyperfine interaction.

Special attention has to be given to the recoil corrections connected with two-photon exchange diagrams shown in Fig.1 in the case of the electron-muon hyperfine interaction. For the singlet-triplet splitting the leading order recoil contribution to the interaction operator between the muon and electron is determined as follows [6, 15, 26]:

$$
\Delta V_{r e c, \mu e, 2 \gamma}^{h f s}\left(\mathbf{x}_{\mu e}\right)=8 \frac{\alpha^{2}}{m_{\mu}^{2}-m_{e}^{2}} \ln \frac{m_{\mu}}{m_{e}} \delta\left(\mathbf{x}_{\mu e}\right) .
$$

Averaging the potential $\Delta V_{r e c, \mu e, 2 \gamma}^{h f s}$ over the wave functions (41) we obtain the leading order recoil correction to the hyperfine splitting:

$$
\Delta \nu_{r e c, \mu e, 2 \gamma}^{h f s}=\nu_{F} \frac{3 \alpha m_{e} m_{\mu}}{\pi\left(m_{\mu}^{2}-m_{e}^{2}\right)} \ln \frac{m_{\mu}}{m_{e}}=0.812 \mathrm{MHz} .
$$

There exist also the two-photon interactions between the bound particles of muonic helium atom when one hyperfine photon transfers the interaction from the electron to muon and another Coulomb photon from the electron to the nucleus (or from the muon to the nucleus). Supposing that these amplitudes give smaller contribution to the hyperfine splitting we include them in the theoretical error.

\section{ELECTRON VERTEX CORRECTIONS}

In the initial approximation the potential of the hyperfine splitting is determined by (3). It leads to the energy splitting of order $\alpha^{4}$. In QED perturbation theory there is the electron vertex correction to the potential (3) which is defined by the diagram in Fig. 2(a). In momentum representation the corresponding operator of hyperfine interaction has the form [22]:

$$
\Delta V_{v e r t e x}^{h f s}\left(k^{2}\right)=-\frac{8 \alpha^{2}\left(1+\kappa_{\mu}\right)}{3 m_{e} m_{\mu}}\left(\frac{\boldsymbol{\sigma}_{e} \boldsymbol{\sigma}_{\mu}}{4}\right)\left[G_{M}^{(e)}\left(k^{2}\right)-1\right],
$$

where $G_{M}^{(e)}\left(k^{2}\right)$ is the electron magnetic form factor. We extracted for the convenience the factor $\alpha / \pi$ from $\left[G_{M}^{(e)}\left(k^{2}\right)-1\right]$. Usually used approximation for the electron magnetic form factor $G_{M}^{(e)}\left(k^{2}\right) \approx G_{M}^{(e)}(0)=1+\kappa_{e}$ is not quite correct in this task. Indeed, characteristic momentum of the exchanged photon is $k \sim \alpha M_{\mu}$. It is impossible to neglect it in the magnetic form factor as compared with the electron mass $m_{e}$. So, we should use exact oneloop expression for the magnetic form factor [27]. Trying to improve the previous estimation 
of the correction due to the electron anomalous magnetic moment we will use further exact one-loop expression for the Pauli form factor $g\left(k^{2}\right)$ known from the QED calculation (see [27]) setting $G_{M}^{(e)}\left(k^{2}\right)-1 \approx g\left(k^{2}\right)$.

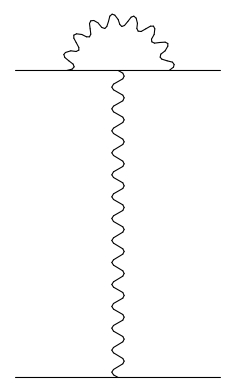

a

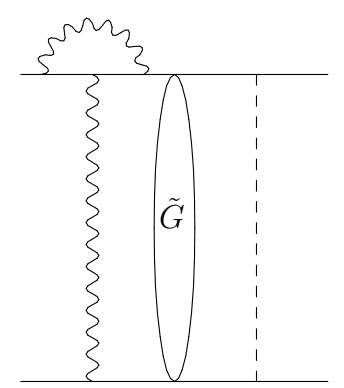

$\mathrm{b}$

FIG. 2: The electron vertex corrections. The dashed line represents the Coulomb photon. The wave line represents the hyperfine part of the Breit potential. $\tilde{G}$ is the reduced Coulomb Green's function.

Using the Fourier transform of the potential (32) and averaging the obtained expression over wave functions (4) we represent the electron vertex correction to the hyperfine splitting as follows [22]:

$$
\Delta \nu_{v e r t}^{h f s}=\nu_{F} \frac{\alpha\left(1+\kappa_{\mu}\right) M_{e}}{2 \pi^{2} M_{\mu}}\left(\frac{m_{e}}{\alpha M_{\mu}}\right)^{3} \int_{0}^{\infty} \frac{k^{2} g\left(k^{2}\right) d k\left(1-3 k^{2} \frac{m_{e}^{2}}{W_{\mu}^{2}}+2 k^{4} \frac{m_{e}^{4}}{W_{\mu}^{4}}\right)}{\left[1+k^{2}\left(\frac{m_{e}^{2}}{W_{\mu}^{2}}\right)\right]^{4}\left[\frac{M_{e}^{2}}{M_{\mu}^{2}}+k^{2}\left(\frac{m_{e}^{2}}{W_{\mu}^{2}}\right)\right]^{2}}=5.078 \mathrm{MHz} .
$$

Let us remark that the contribution (33) is of order $\alpha^{5}$. Numerical value (33) is obtained after numerical integration with the exact one-loop expression of the Pauli electron form factor $g\left(k^{2}\right)$ [27]. If we use the value $g\left(k^{2}=0\right)$ then the electron vertex correction is equal $5.246 \mathrm{MHz}$. So, using the exact expression of the electron form factor $g\left(k^{2}\right)$ in the one-loop approximation we observe small decrease of the vertex correction to the hyperfine splitting from $1 \gamma$ interaction. Taking the expression (32) as an additional perturbation potential we have to calculate its contribution to HFS in second order perturbation theory (see the diagram in Fig. 2(b)). In this case the dashed line represents the Coulomb Hamiltonian $\Delta H$ (2). Following the method of the calculation formulated in previous section we divide again total contribution from the amplitude in Fig. 2(b) into several parts which correspond to the muon ground state $(n=2)$ and muon excited intermediate states $(n \neq 2)$. In this way first contribution with $n=2 S$ takes the form:

$\Delta \nu_{v e r t, 2 S}^{h f s}=\frac{8 \alpha^{2}\left(1+\kappa_{\mu}\right)}{3 \pi^{2} m_{e} m_{\mu}} \int_{0}^{\infty} k g\left(k^{2}\right) d k \int d \mathbf{x}_{1} \int d \mathbf{x}_{3} \psi_{e}\left(\mathbf{x}_{3}\right) \Delta V_{1}\left(k, \mathbf{x}_{3}\right) G_{e}\left(\mathbf{x}_{1}, \mathbf{x}_{3}\right) \Delta V_{\mu}\left(\mathbf{x}_{1}\right) \psi_{e}\left(\mathbf{x}_{1}\right)$,

where $\Delta V_{\mu}\left(\mathbf{x}_{1}\right)$ is defined by (9) and

$$
\Delta V_{1}\left(k, \mathbf{x}_{3}\right)=\int d \mathbf{x}_{4} \psi_{\mu}\left(\mathbf{x}_{4}\right) \frac{\sin \left(k\left|\mathbf{x}_{3}-\mathbf{x}_{4}\right|\right)}{\left|\mathbf{x}_{3}-\mathbf{x}_{4}\right|} \psi_{\mu}\left(\mathbf{x}_{4}\right)=\frac{\sin \left(k x_{3}\right)}{x_{3}} \frac{\left(1-3 \frac{k^{2}}{W_{\mu}^{2}}+2 \frac{k^{4}}{W_{\mu}^{4}}\right)}{\left[1+\frac{k^{2}}{W_{\mu}^{2}}\right]^{4}} .
$$


Substituting the electron Green's function (8) in (34) we perform all coordinate integrations analytically and write (34) in integral form $\left(a_{1}=k m_{e} / W_{\mu}\right)$ :

$$
\begin{gathered}
\Delta \nu_{v e r t, 2 S}^{h f s}=\nu_{F} \frac{\alpha\left(1+\kappa_{\mu}\right)}{2 \pi^{2}}\left(\frac{m_{e}}{\alpha M_{\mu}}\right)^{2}\left(\frac{M_{e}}{M_{\mu}}\right) \int_{0}^{\infty} \frac{k g\left(k^{2}\right) a_{1}^{4}\left(1-3 a_{1}^{2} k^{2}+2 a_{1}^{4} k^{4}\right) d k}{\left(1+a_{1}^{2}\right)^{8}\left(a_{1}^{2}+M_{e}^{2} / M_{\mu}^{2}\right)^{3}} \times \\
{\left[a_{1}\left(2 a_{1}^{6}+8 a_{1}^{5}+8 a_{1}^{2}+14\right)+\frac{M_{e}}{M_{\mu}}\left(-2 a_{1}^{7}-4 a_{1}^{5}+14 a_{1}+14\left(a_{1}^{2}-1\right)^{4} \arctan \frac{1}{a_{1}}\right)\right]=0.067 \mathrm{MHz},}
\end{gathered}
$$

where we expand also the integral function in the numerator in small parameter $M_{e} / M_{\mu}$ and carry out last integration numerically.

Second part of the vertex contribution (Fig! $2(\mathrm{~b}))$ with $n=2 P$ has the following general form:

$$
\begin{gathered}
\Delta \nu_{v e r t, 2 P}^{h f s}=\frac{8 \alpha^{2}\left(1+\kappa_{\mu}\right)}{3 \pi^{2} m_{e} m_{\mu}} \int_{0}^{\infty} k g\left(k^{2}\right) d k \int d \mathbf{x}_{1} \int d \mathbf{x}_{2} \int d \mathbf{x}_{3} \int d \mathbf{x}_{4} \psi_{\mu}^{*}\left(\mathbf{x}_{4}\right) \psi_{e}^{*}\left(\mathbf{x}_{3}\right) \times \\
\sum_{m=-1}^{1} \psi_{\mu, 2 P, m}\left(\mathbf{x}_{4}\right) \psi_{\mu, 2 P, m}^{*}\left(\mathbf{x}_{2}\right) \frac{\sin k\left|\mathbf{x}_{4}-\mathbf{x}_{3}\right|}{\left|\mathbf{x}_{4}-\mathbf{x}_{3}\right|} \sum_{n \neq 0} \frac{\psi_{e, n}\left(\mathbf{x}_{3}\right) \psi_{e, n}^{*}\left(\mathbf{x}_{1}\right)}{E_{e 0}-E_{e, n}} \Delta H\left(\mathbf{x}_{1}, \mathbf{x}_{2}\right) \psi_{\mu}\left(\mathbf{x}_{2}\right) \psi_{e}\left(\mathbf{x}_{1}\right) .
\end{gathered}
$$

Then we make two subsequent integrations over coordinates $\mathbf{x}_{2}$ and $\mathbf{x}_{4}$ :

$$
\begin{gathered}
\delta \tilde{V}_{2}\left(\mathbf{x}_{1}\right)=\int d \mathbf{x}_{2} x_{2} e^{-W_{\mu} x_{2}}\left(1-\frac{W_{\mu} x_{2}}{2}\right) \frac{\alpha\left(\mathbf{n}_{2} \mathbf{n}_{4}\right)}{\left|\mathbf{x}_{2}-\mathbf{x}_{1}\right|}= \\
=\frac{2 \pi \alpha}{W_{\mu}^{3}} \frac{e^{-x_{1}}\left(\mathbf{n}_{1} \mathbf{n}_{4}\right)}{x_{1}^{2}}\left[x_{1}^{4}+4 x_{1}^{3}+12 x_{1}^{2}+24 x_{1}-24 e^{x_{1}}+24\right] \\
\delta \tilde{V}_{1}\left(\mathbf{x}_{3}\right)=\int d \mathbf{x}_{4} x_{4} e^{-W_{\mu} x_{4}}\left(1-\frac{W_{\mu} x_{4}}{2}\right)\left(\mathbf{n}_{1} \mathbf{n}_{4}\right) \frac{\sin k\left|\mathbf{x}_{4}-\mathbf{x}_{3}\right|}{\left|\mathbf{x}_{4}-\mathbf{x}_{3}\right|}= \\
=\frac{48 \pi}{W_{\mu}^{3}}\left(\mathbf{n}_{1} \mathbf{n}_{3}\right) \frac{\left(k x_{3} \cos k x_{3}-\sin k x_{3}\right)\left(1-\frac{k^{2}}{W_{\mu}^{2}}\right)}{x_{3}^{2}\left(1+\frac{k^{2}}{W_{\mu}^{2}}\right)^{4}} .
\end{gathered}
$$

Finally, after analytical integration over coordinates $\mathbf{x}_{1}$ and $\mathbf{x}_{3}$ with the Green's function (12) we present the correction (37) in the form:

$$
\begin{gathered}
\Delta \nu_{\text {vert }, 2 P}^{h f s}=-\nu_{F} \frac{3 \alpha\left(1+\kappa_{\mu}\right) M_{e} m_{e}^{2}}{\pi^{2} M_{\mu}\left(\alpha M_{\mu}\right)^{2}} \int_{0}^{\infty} \frac{k g\left(k^{2}\right) a_{1}^{3}\left(1-a_{1}^{2}\right) d k}{\left(a_{1}^{2}+1\right)^{4}\left(1+\frac{M_{e}}{M_{\mu}}\right)^{5}\left(a_{1}^{2}+\frac{M_{e}^{2}}{M_{\mu}^{2}}\right)^{2}\left[a_{1}^{2}+\left(1+\frac{M_{e}}{M_{\mu}}\right)^{2}\right]_{(40)}^{4}} \\
\times\left\{12-12 a_{1}^{2}+\frac{M_{e}}{M_{\mu}}\left[75 a_{1}^{8}+215 a_{1}^{6}+209 a_{1}^{4}+9 a_{1}^{2}+15 \pi a_{1}\left(1+a_{1}^{2}\right)^{4}-75\left(1+a_{1}^{2}\right)^{4} a_{1} \arctan a_{1}+156\right]\right\}= \\
=-0.309 \mathrm{MHz} .
\end{gathered}
$$

The expansion of integral function in the numerator of (40) is used up to terms of order $O\left(M_{e} / M_{\mu}\right)$. In order to evaluate the vertex contribution due to excited states of the muon 
we replace as above in (15) exact Green's function of the electron by free Green's function. Then our starting point for the calculation is related with the following expression:

$$
\begin{aligned}
\Delta \nu_{v e r t, n \neq 2}^{h f s}= & -\frac{8 \alpha^{2}\left(1+\kappa_{\mu}\right)}{3 \pi^{2} m_{e} m_{\mu}} \int_{0}^{\infty} k g\left(k^{2}\right) d k \int d \mathbf{x}_{3} \int d \mathbf{x}_{4} \psi_{\mu}^{*}\left(\mathbf{x}_{4}\right) \psi_{e}^{*}\left(\mathbf{x}_{3}\right) \frac{\sin k\left|\mathbf{x}_{4}-\mathbf{x}_{3}\right|}{\left|\mathbf{x}_{4}-\mathbf{x}_{3}\right|} \times \\
& \int d \mathbf{x}_{1} \int d \mathbf{x}_{2} \sum_{n \neq 2} \psi_{\mu, n}\left(\mathbf{x}_{4}\right) \psi_{\mu, n}^{*}\left(\mathbf{x}_{2}\right) \frac{e^{-b\left|\mathbf{x}_{3}-\mathbf{x}_{1}\right|}}{\left|\mathbf{x}_{3}-\mathbf{x}_{1}\right|\left|\mathbf{x}_{2}-\mathbf{x}_{1}\right|} \psi_{\mu}\left(\mathbf{x}_{2}\right) \psi_{e}\left(\mathbf{x}_{1}\right) .
\end{aligned}
$$

The integration over $\mathbf{x}_{1}$ is done in (16). The term proportional to $1 / b$ does not contribute because of the orthogonality of wave functions. The contribution of second term in square brackets in (16) can be transformed initially by means of completeness condition:

$$
\begin{gathered}
\Delta \nu_{v e r t, n \neq 2}^{h f s}=\frac{8 \alpha^{3}\left(1+\kappa_{\mu}\right) M_{e}}{3 \pi^{2} m_{e} m_{\mu}} \frac{W_{e}^{3 / 2}}{\sqrt{\pi}} \int_{0}^{\infty} k g\left(k^{2}\right) d k \int d \mathbf{x}_{3} \int d \mathbf{x}_{4} \psi_{\mu}^{*}\left(\mathbf{x}_{4}\right) \psi_{e}^{*}\left(\mathbf{x}_{3}\right) \frac{\sin k\left|\mathbf{x}_{4}-\mathbf{x}_{3}\right|}{\left|\mathbf{x}_{4}-\mathbf{x}_{3}\right|} \times \\
\int d \mathbf{x}_{2}\left[\delta\left(\mathbf{x}_{2}-\mathbf{x}_{3}\right)-\psi_{\mu, 2 S}\left(\mathbf{x}_{3}\right) \psi_{\mu, 2 S}^{*}\left(\mathbf{x}_{2}\right)-\sum_{m=-1}^{1} \psi_{\mu, 2 P, m}\left(\mathbf{x}_{3}\right) \psi_{\mu, 2 P, m}^{*}\left(\mathbf{x}_{2}\right)\right]\left|\mathbf{x}_{3}-\mathbf{x}_{2}\right| \psi_{\mu}\left(\mathbf{x}_{2}\right) .
\end{gathered}
$$

Then the integration over particle coordinates is performed analytically in all terms in (42). Remaining integration over $k$ is carried out numerically. Omitting intermediate expressions for numerous integrals which are calculated in the same way as in previous corrections, we present final numerical result

$$
\Delta \nu_{\text {vert }, n \neq 2}^{h f s}=0.992 M H z
$$

The electron vertex corrections investigated in this section have the order $\alpha^{5}$ in the hyperfine interval. Summary value of all obtained contributions in second order PT is equal to $0.750 \mathrm{MHz}$. Summing this number with the correction (33) we obtain the value 5.828 $\mathrm{MHz}$. It differs by a significant value $0.582 \mathrm{MHz}$ from the result $5.246 \mathrm{MHz}$ which was used previously by many authors for the estimation of the electron anomalous magnetic moment contribution (see [8, 14, 15].

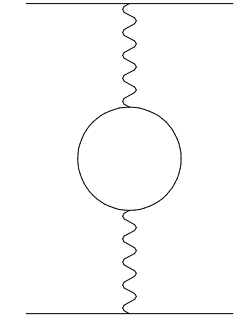

a

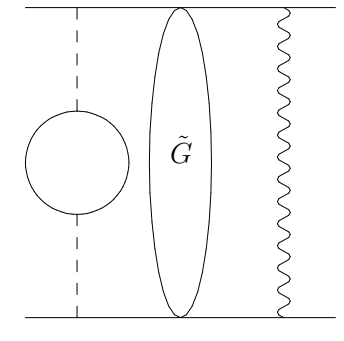

$\mathrm{b}$

FIG. 3: Vacuum polarization effects. The dashed line represents the Coulomb photon. The wave line represents the hyperfine part of the Breit potential. $\tilde{G}$ is the reduced Coulomb Green's function. 


\section{EFFECTS OF VACUUM POLARIZATION}

The vacuum effects change significantly the interaction (11)-(2) between particles in muonic helium atom. One of the most important contributions is related with the vacuum polarization (VP). The value of the electron vacuum polarization corrections to the hyperfine splitting is determined by the parameter equal to the ratio of the Compton wave length of the electron and the radius of the Bohr orbit in the subsystem $\left(\mu_{2}^{4} \mathrm{He}\right)^{+}: M_{\mu} \alpha / m_{e}=$ $1.46727 \ldots$... It is impossible to use the expansion in $\alpha$ for such contributions. So, we calculate them performing the analytical and numerical integration over the particle coordinates and other parameters. The effect of the electron vacuum polarization leads to the appearance of a number of additional terms in the Hamiltonian operator describing $(e-\alpha)-,(\mu-\alpha)$ - and $(e-\mu)$-interactions which we present in the form [20, 22, 27]:

$$
\begin{gathered}
\Delta V_{V P}^{e \alpha}\left(x_{e}\right)=\frac{\alpha}{3 \pi} \int_{1}^{\infty} d \xi \rho(\xi)\left(-\frac{2 \alpha}{x_{e}}\right) e^{-2 m_{e} \xi x_{e}}, \rho(\xi)=\frac{\sqrt{\xi^{2}-1}\left(2 \xi^{2}+1\right)}{\xi^{4}} \\
\Delta V_{V P}^{\mu \alpha}\left(x_{\mu}\right)=\frac{\alpha}{3 \pi} \int_{1}^{\infty} d \xi \rho(\xi)\left(-\frac{2 \alpha}{x_{\mu}}\right) e^{-2 m_{e} \xi x_{\mu}} \\
\Delta V_{V P}^{e \mu}\left(\left|\mathbf{x}_{e}-\mathbf{x}_{\mu}\right|\right)=\frac{\alpha}{3 \pi} \int_{1}^{\infty} d \xi \rho(\xi) \frac{\alpha}{x_{e \mu}} e^{-2 m_{e} \xi x_{e \mu}}
\end{gathered}
$$

where $x_{e \mu}=\left|\mathbf{x}_{e}-\mathbf{x}_{\mu}\right|$. They give contributions in second order perturbation theory and are discussed below. In first order perturbation theory the contribution of the vacuum polarization is connected with the modification of the hyperfine splitting part of the Hamiltonian (3) (the diagram in Fig,2(a)). It is described by the expression [18, 19]:

$$
\Delta V_{V P}^{h f s}\left(\mathbf{x}_{e \mu}\right)=-\frac{8 \pi \alpha\left(1+\kappa_{\mu}\right)}{3 m_{e} m_{\mu}} \frac{\boldsymbol{\sigma}_{1} \boldsymbol{\sigma}_{2}}{4} \frac{\alpha}{3 \pi} \int_{1}^{\infty} \rho(\xi) d \xi\left[\pi \delta\left(\mathbf{x}_{\mathbf{e} \mu}\right)-\frac{m_{e}^{2} \xi^{2}}{x_{e \mu}} e^{-2 m_{e} \xi x_{e \mu}}\right] .
$$

Averaging the potential (47) over the wave function (41) we obtain the following expression:

$$
\begin{gathered}
\Delta \nu_{V P, e-\mu}^{h f s,(1)}=\frac{\alpha^{2}\left(1+\kappa_{\mu}\right)}{9 m_{e} m_{\mu}} \frac{W_{e}^{3} W_{\mu}^{3}}{\pi^{2}} \int_{1}^{\infty} \rho(\xi) d \xi \int d \mathbf{x}_{e} \int d \mathbf{x}_{\mu} e^{-W_{\mu} x_{\mu}}\left(1-\frac{W_{\mu} x_{\mu}}{2}\right)^{2} \times \\
e^{-2 W_{e} x_{e}}\left[\pi \delta\left(\mathbf{x}_{\mu}-\mathbf{x}_{e}\right)-\frac{m_{e}^{2} \xi^{2}}{\left|\mathbf{x}_{\mu}-\mathbf{x}_{e}\right|}\right] e^{-2 m_{e} \xi\left|\mathbf{x}_{\mu}-\mathbf{x}_{e}\right|}
\end{gathered}
$$

where the superscript (1) designates the correction of first order PT and the subscript $e-\mu$ corresponds to the electron-muon interaction. There are two integrals over the muon and electron coordinates in (48) which can be calculated analytically. As a result the correction (48) takes the form of one-dimensional integral in the parameter $\xi\left(a_{2}=m_{e} \xi / M_{\mu} \alpha\right)$ :

$$
\begin{aligned}
& \Delta \nu_{V P, e-\mu}^{h f s,(1)}=\nu_{F} \frac{\alpha\left(1+\kappa_{\mu}\right) M_{e}}{6 \pi M_{\mu}\left(1+\frac{M_{e}}{M_{\mu}}\right)^{5}} \int_{1}^{\infty} \frac{\rho(\xi) d \xi}{\left(1+a_{2}\right)^{4}\left(a_{2}+\frac{M_{e}}{M_{\mu}}\right)^{2}}\left\{a_{2}^{4}\left[1+\frac{M_{e}}{M_{\mu}}\left(4 \frac{M_{e}}{M_{\mu}}-1\right)\right]+\right. \\
& \quad+2 a_{2}^{3}\left(2+\frac{M_{e}}{M_{\mu}}\right)\left[1+\frac{M_{e}}{M_{\mu}}\left(4 \frac{M_{e}}{M_{\mu}}-1\right)\right]+a_{2}^{2}\left(1+\frac{M_{e}}{M_{\mu}}\right)\left[4+\frac{M_{e}}{M_{\mu}}\left(11 \frac{M_{e}}{M_{\mu}}-12\right)\right]+
\end{aligned}
$$




$$
\left.+4 a_{2}\left[1+\frac{M_{e}}{M_{\mu}}-\frac{M_{e}^{2}}{M_{\mu}^{2}}+2 \frac{M_{e}^{3}}{M_{\mu}^{3}}\right]+2 \frac{M_{e}}{M_{\mu}}\left[1+\frac{M_{e}}{M_{\mu}}\left(\frac{M_{e}}{M_{\mu}}-1\right)\right]\right\}=0.012 M H z .
$$

In the leading order this contribution has fifth order in $\alpha$ and first order in the electron-muon recoil parameter $M_{e} / M_{\mu}$. The similar contribution of the muon vacuum polarization to the hyperfine splitting is extremely small $\left(\sim 10^{-6} \mathrm{MHz}\right)$.

Let us consider corrections of the electron vacuum polarization (44)-(46) in the second order perturbation theory (the diagram in Fig. 2(b)). The contribution of the operator (44) to the hyperfine splitting can be written as follows:

$$
\begin{gathered}
\Delta \nu_{V P, e \alpha}^{h f s,(2)}=\frac{32 \alpha^{3}}{9 m_{e} m_{\mu}} \int d \mathbf{x}_{1} \int d \mathbf{x}_{2} \int d \mathbf{x}_{3} \int_{1}^{\infty} \rho(\xi) d \xi \psi_{\mu}^{*}\left(\mathbf{x}_{3}\right) \psi_{e}^{*}\left(\mathbf{x}_{3}\right) \times \\
\sum_{n, m}^{\prime} \frac{\psi_{\mu, n}\left(\mathbf{x}_{3}\right) \psi_{e, m}\left(\mathbf{x}_{3}\right) \psi_{\mu, n}^{*}\left(\mathbf{x}_{2}\right) \psi_{e, m}^{*}\left(\mathbf{x}_{1}\right)}{E_{\mu}+E_{e}-E_{\mu, n}-E_{e, m}} \frac{e^{-2 m_{e} \xi x_{1}}}{x_{1}} \psi_{\mu}\left(\mathbf{x}_{2}\right) \psi_{e}\left(\mathbf{x}_{1}\right)
\end{gathered}
$$

Here the summation is carried out over the complete system of the electron and muon eigenstates excluding the state $1 s_{1 / 2}^{(e)} 2 s_{1 / 2}^{(\mu)}$. Single contribution to the sum in (150) is determined by the $2 S$ muon intermediate state because of the wave function orthogonality condition:

$$
\Delta \nu_{V P, e \alpha}^{h f s,(2)}=\frac{32 \alpha^{3}\left(1+\kappa_{\mu}\right)}{9 m_{e} m_{\mu}} \int_{1}^{\infty} \rho(\xi) d \xi \int d \mathbf{x}_{1} \int d \mathbf{x}_{3}\left|\psi_{\mu}\left(\mathbf{x}_{3}\right)\right|^{2} \psi_{e}\left(\mathbf{x}_{3}\right) G_{e}\left(\mathbf{x}_{1}, \mathbf{x}_{3}\right) \frac{e^{-2 m_{e} \xi x_{1}}}{x_{1}} \psi_{e}\left(\mathbf{x}_{1}\right) .
$$

Using the electron Green's function (8) we perform analytical integration over coordinates $\mathbf{x}_{1}$ and $\mathbf{x}_{3}$ and present (51) as follows:

$$
\Delta \nu_{V P, e \alpha}^{h f s,(2)}=-\nu_{F} \frac{\alpha\left(1+\kappa_{\mu}\right) M_{e}}{3 \pi M_{\mu}} \int_{1}^{\infty} \rho(\xi) d \xi \frac{4+a_{2}\left(a_{2}+2\right)^{2}}{a_{2}\left(a_{2}+1\right)^{4}}=-0.028 \mathrm{MHz} .
$$

This contribution has the same order of the magnitude $O\left(\alpha^{5} M_{e} / M_{\mu}\right)$ as the previous one in first order perturbation theory. The similar calculation can be performed in the case of muon-nucleus vacuum polarization operator (45). The intermediate electron state is the $1 \mathrm{~S}$ state and the reduced Coulomb Green's function of the system transforms to the Green's function of the muon. The correction of the operator (45) to the hyperfine splitting is obtained in the following integral form:

$$
\Delta \nu_{V P, \mu \alpha}^{h f s,(2)}=\frac{32 \alpha^{3}\left(1+\kappa_{\mu}\right)}{9 m_{e} m_{\mu}} \int_{1}^{\infty} \rho(\xi) d \xi \int d \mathbf{x}_{2} \int d \mathbf{x}_{3} \psi_{\mu}^{*}\left(\mathbf{x}_{3}\right) \psi_{\mu}\left(\mathbf{x}_{2}\right)\left|\psi_{e}\left(\mathbf{x}_{3}\right)\right|^{2} \frac{e^{-2 m_{e} \xi x_{2}}}{x_{2}} G_{\mu}\left(\mathbf{x}_{2}, \mathbf{x}_{3}\right),
$$

where

$$
\begin{gathered}
G_{\mu}\left(\mathbf{x}_{2}, \mathbf{x}_{3}\right)=-\frac{\alpha M_{\mu}^{2}}{16 \pi} \frac{e^{-\left(x_{2}+x_{3}\right) / 2}}{x_{2} x_{3}}\left[8 x_{<}-4 x_{<}^{2}+8 x_{>}+12 x_{<} x_{>}-26 x_{<}^{2} x_{>}+2 x_{<}^{3} x_{>}-4 x_{>}^{2}-(54)\right. \\
-26 x_{<} x_{>}^{2}+23 x_{<}^{2} x_{>}^{2}-x_{<}^{3} x_{>}^{2}+2 x_{<} x_{>}^{3}-x_{<}^{2} x_{>}^{3}+4 e^{x}\left(1-x_{<}\right)\left(x_{>}-2\right) x_{>}+4\left(x_{<}-2\right) x_{<}\left(x_{>}-2\right) x_{>} \times \\
{\left[-2 C+E i\left(x_{<}\right)-\ln \left(x_{<}\right)-\ln \left(x_{>}\right)\right] .}
\end{gathered}
$$

Integrating over coordinates we obtain:

$$
\Delta \nu_{V P, \mu \alpha}^{h f s,(2)}=-\nu_{F} \frac{\alpha\left(1+\kappa_{\mu}\right) M_{e}}{2 \pi M_{\mu}} \int_{1}^{\infty} \rho(\xi) d \xi \frac{4 a_{2}^{4}+8 a_{2}^{3}+4 a_{2}^{2}+6 a_{2}+1}{\left(1+a_{2}\right)^{6}}=-0.099 \mathrm{MHz} .
$$


The vacuum polarization correction connected with the operator (46) in the second order perturbation theory is the most difficult for the calculation. Indeed, in this case we have to consider the intermediate excited states both for the muon and electron. We have divided total contribution into several parts. The first part in which the intermediate muon is in the $2 \mathrm{~S}$ state can be written as:

$\Delta \nu_{V P, \mu e, 2 S}^{h f s,(2)}=-\nu_{F} \frac{\alpha^{3}\left(1+\kappa_{\mu}\right) M_{e}^{2}}{3 \pi W_{\mu}^{2}} \int_{1}^{\infty} \rho(\xi) d \xi \int_{0}^{\infty} x_{1}^{2} e^{-\frac{M_{e}}{M_{\mu}} x_{1}} d x_{1} \int_{0}^{\infty} x_{3}^{2} d x_{3}\left(1-\frac{x_{3}}{2}\right)^{2} e^{-x_{3}\left(1+\frac{M_{e}}{M_{\mu}}\right)}$

$\times \Delta V_{V P, \mu}\left(x_{1}\right)\left[\frac{1}{\frac{M_{e}}{M_{\mu}} x_{>}}-\ln \frac{M_{e}}{M_{\mu}} x_{>}-\ln \frac{M_{e}}{M_{\mu}} x_{<}+E i\left(\frac{M_{e}}{M_{\mu}} x_{<}\right)+\frac{7}{2}-2 C-\frac{M_{e}\left(x_{1}+x_{3}\right)}{2 M_{\mu}}+\frac{1-e^{\frac{M_{e}}{M_{\mu}}} x_{<}}{\frac{M_{e}}{M_{\mu}} x_{<}}\right]$,

where the function $V_{V P \mu}\left(x_{1}\right)$ is equal to

$$
\begin{gathered}
\Delta V_{V P, \mu}\left(x_{1}\right)=\int_{0}^{\infty} x_{2}^{2} e^{-x_{2}}\left(1-\frac{x_{2}}{2}\right)^{2} d x_{2} \int_{1}^{1} d z \frac{e^{-a_{2}\left|\mathbf{x}_{1}-\mathbf{x}_{2}\right|}}{\left|\mathbf{x}_{1}-\mathbf{x}_{2}\right|}= \\
=\frac{1}{2 x_{1}\left(a_{2}^{2}-1\right)^{4}}\left\{8 e^{-a_{2} x_{1}}\left(2 a_{2}^{4}+3 a_{2}^{2}+1\right)+e^{-x_{1}}\left[a_{2}^{6} x_{1}\left(x_{1}-2\right)^{2}+a_{2}^{4}\left(x_{1}\left(10-3\left(x_{1}-2\right) x_{1}\right)-16\right)+\right.\right. \\
\left.\left.\left.+a_{2}^{2}\left(3 x_{1}^{3}-8 x_{1}-24\right)-x_{1}\left(x_{1}\left(x_{1}+2\right)+6\right)-8\right)\right]\right\} .
\end{gathered}
$$

Substituting (57) in (58) we obtain the following result with an accuracy $O\left(M_{e} / M_{\mu}\right)$ :

$$
\begin{gathered}
\Delta \nu_{V P, \mu e, 2 S}^{h f s,(2)}=-\nu_{F} \frac{\alpha M_{e}\left(1+\kappa_{\mu}\right)}{768 \pi M_{\mu}} \int_{1}^{\infty} \frac{\rho(\xi) d \xi}{a_{2}\left(1+a_{2}\right)^{8}}\left(77 a_{2}^{7}+616 a_{2}^{6}+2151 a_{2}^{5}+4272 a_{2}^{4}+\right. \\
\left.+5267 a_{2}^{3}+4168 a_{2}^{2}+1929 a_{2}+512\right)=-0.009 \text { MHz. }
\end{gathered}
$$

The second part of the vacuum polarization correction to the hyperfine splitting due to the $2 P$ muon state can be presented as

$$
\begin{aligned}
\Delta \nu_{V P, \mu e, 2 P}^{h f s,(2)}= & \frac{16 \alpha^{3}\left(1+\kappa_{\mu}\right)}{9 m_{e} m_{\mu}} \int d \mathbf{x}_{1} \int d \mathbf{x}_{2} \int d \mathbf{x}_{3} \psi_{\mu}^{*}\left(\mathbf{x}_{3}\right) \psi_{e}^{*}\left(\mathbf{x}_{3}\right) \sum_{m=-1}^{1} \psi_{\mu, 2 P, m}\left(\mathbf{x}_{3}\right) \psi_{\mu, 2 P, m}^{*}\left(\mathbf{x}_{2}\right) \times \\
& \times \sum_{n \neq 0} \frac{\psi_{e, n}\left(\mathbf{x}_{3}\right) \psi_{e, n}^{*}\left(\mathbf{x}_{1}\right)}{E_{e}-E_{e, n}} \int_{1}^{\infty} \rho(\xi) d \xi \frac{e^{-2 m_{e} \xi\left|\mathbf{x}_{2}-\mathbf{x}_{1}\right|}}{\left|\mathbf{x}_{2}-\mathbf{x}_{1}\right|} \psi_{\mu}\left(\mathbf{x}_{2}\right) \psi_{e}\left(\mathbf{x}_{1}\right) .
\end{aligned}
$$

Averaging over the directions of the vector $\mathbf{n}_{3}<\left(\mathbf{n}_{3} \mathbf{n}_{2}\right)\left(\mathbf{n}_{3} \mathbf{n}_{1}\right)>=\left(\mathbf{n}_{1} \mathbf{n}_{2}\right) / 3$ and evaluating the integral

$$
\begin{gathered}
\Delta \tilde{V}_{V P, \mu}\left(x_{1}\right)=\int_{0}^{\infty} x_{2}^{3} e^{-x_{2}}\left(1-\frac{x_{2}}{2}\right) d x_{2} \int_{1}^{1} z d z \frac{e^{-a_{2}\left|\mathbf{x}_{1}-\mathbf{x}_{2}\right|}}{\left|\mathbf{x}_{1}-\mathbf{x}_{2}\right|}=\frac{1}{x_{1}^{2}\left(a_{2}^{2}-1\right)^{4}}\left\{e^{-x_{1}} \times\right. \\
\left.\left[24\left(a_{2}^{2}+1\right)\left(x_{1}+1\right)-12 x_{1}^{2}\left(a_{2}^{4}-1\right)+2 x_{1}^{3}\left(a_{2}^{6}-3 a_{2}^{2}+2\right)-x_{1}^{4}\left(a_{2}^{2}-1\right)^{3}\right]-24 e^{-a_{2} x_{1}}\left(a_{2}^{2}+1\right)\left(a_{2} x_{1}+1\right)\right\},
\end{gathered}
$$

we find this contribution numerically:

$$
\Delta \nu_{V P, \mu e, 2 P}^{h f s,(2)}=-\nu_{F} \frac{9 \alpha\left(1+\kappa_{\mu}\right) M_{e}}{192 \pi M_{\mu}} \int_{1}^{\infty} \frac{\rho(\xi) d \xi}{\left(1+a_{2}\right)^{8}}\left[5 a_{2}^{6}+40 a_{2}^{5}+139 a_{2}^{4}+272 a_{2}^{3}+\right.
$$




$$
\left.+323 a_{2}^{2}+232 a_{2}+93\right]=-0.019 M H z .
$$

Finally, there exists the contribution of the muon intermediate states with $n \neq 2 S, 2 P$ :

$$
\begin{aligned}
\Delta \nu_{n \neq 2 S, 2 P}^{h f s,(2)}= & -\frac{16 \alpha^{3}\left(1+\kappa_{\mu}\right)}{9 m_{e} m_{\mu}} \int d \mathbf{x}_{1} \int d \mathbf{x}_{2} \int d \mathbf{x}_{3} \psi_{\mu}^{*}\left(\mathbf{x}_{3}\right) \psi_{e}^{*}\left(\mathbf{x}_{3}\right) \sum_{n \neq 2} \psi_{\mu, n}\left(\mathbf{x}_{3}\right) \psi_{\mu, n}^{*}\left(\mathbf{x}_{2}\right) \times \\
& G_{e}\left(\mathbf{x}_{1}, \mathbf{x}_{3}, E_{\mu}+E_{e}-E_{\mu, n}\right) \int_{1}^{\infty} \rho(\xi) d \xi \frac{e^{-2 m_{e} \xi\left|\mathbf{x}_{2}-\mathbf{x}_{1}\right|}}{\left|\mathbf{x}_{2}-\mathbf{x}_{1}\right|} \psi_{\mu}\left(\mathbf{x}_{2}\right) \psi_{e}\left(\mathbf{x}_{1}\right) .
\end{aligned}
$$

Then we have replaced in (62) exact electron Coulomb Green's function $G_{e}$ by free electron Green's function (15) which contains the parameter $b=\left[2 M_{e}\left(E_{\mu, n}-E_{\mu}-E_{e}\right]^{1 / 2}\right.$. We also replace the electron wave functions by their values at the origin neglecting higher order recoil corrections. After that the integration over $\mathbf{x}_{1}$ can be done analytically:

$$
\begin{gathered}
I_{2}=\int d \mathbf{x}_{1} \frac{e^{-b\left|\mathbf{x}_{3}-\mathbf{x}_{1}\right|}}{\left|\mathbf{x}_{3}-\mathbf{x}_{1}\right|} \frac{e^{-2 m_{e} \xi\left|\mathbf{x}_{2}-\mathbf{x}_{1}\right|}}{\left|\mathbf{x}_{2}-\mathbf{x}_{1}\right|}=-\frac{4 \pi}{W_{\mu}} \frac{e^{-b_{1}\left|\mathbf{x}_{3}-\mathbf{x}_{2}\right|}-e^{-a_{2}\left|\mathbf{x}_{3}-\mathbf{x}_{2}\right|}}{\left|\mathbf{x}_{3}-\mathbf{x}_{2}\right|\left(b_{1}^{2}-a_{2}^{2}\right)}= \\
=-\frac{4 \pi}{W_{\mu}}\left[\frac{1-e^{-a_{2}\left|\mathbf{x}_{3}-\mathbf{x}_{2}\right|}}{a_{2}^{2}\left|\mathbf{x}_{3}-\mathbf{x}_{2}\right|}-\frac{2 b_{1}}{a_{2}^{2}} \ldots\right]
\end{gathered}
$$

where we have performed small-parameter $b_{1}=b / W_{\mu}$ expansion. For the further transformation we use the completeness relation (17) dividing total correction (62) into three terms. The coordinate integration in each of the terms can be presented individually in analytical form. As a result the correction (62) is reduced to one-dimensional integral over the parameter $\xi$ which is evaluated with high accuracy numerically:

$$
\begin{aligned}
\Delta \nu_{n \neq 2 S, 2 P}^{h f s,(2)} & =\nu_{F} \frac{\alpha\left(1+\kappa_{\mu}\right) M_{e}}{384 \pi M_{\mu}} \int_{1}^{\infty} \frac{\rho(\xi) d \xi}{\left(1+a_{2}\right)^{7}}\left(256 a_{2}^{6}+1731 a_{2}^{5}+4949 a_{2}^{4}+\right. \\
& \left.+7686 a_{2}^{3}+6874 a_{2}^{2}+3375 a_{2}+665\right)=0.076 \mathrm{MHz} .
\end{aligned}
$$

We preserve in (64) only leading order term in the ratio $M_{e} / M_{\mu}$. In whole, in (64) we have second order correction in two small parameters $\alpha$ and $M_{e} / M_{\mu}$. We keep in (63) only first term in the square brackets because other terms give recoil corrections of higher order. Numerical values of obtained corrections are presented in Table I.

There exists another contribution of the second order perturbation theory in which we have the vacuum polarization perturbation connected with the hyperfine splitting part of the Breit potential (11) (see Fig. 5). Other perturbation is determined by $\Delta H$ (2). We can divide this correction into three parts. One part corresponds to the muon in the $2 S$-state. The $\delta$-function term in (47) gives the following contribution at $n=2$ (compare with (10)):

$$
\Delta \nu_{V P, 11,2 S}^{h f s,(2)}=\nu_{F}\left(1+\kappa_{\mu}\right) \frac{\alpha}{3 \pi} \int_{1}^{\infty} \rho(\xi) d \xi \frac{631 M_{e}}{256 M_{\mu}} .
$$

Obviously this integral is divergent. So, we have to consider the contribution of second term in (47) to the hyperfine splitting which is determined by more complicated expression:

$$
\Delta \nu_{V P, 12,2 S}^{h f s,(2)}=-\frac{16 \alpha^{2}\left(1+\kappa_{\mu}\right) m_{e}^{2}}{9 \pi m_{e} m_{\mu}} \int_{1}^{\infty} \rho(\xi) \xi^{2} d \xi \int d \mathbf{x}_{4}\left|\psi_{\mu}\left(\mathbf{x}_{4}\right)\right|^{2} \int d \mathbf{x}_{3} \psi_{e}\left(\mathbf{x}_{3}\right) \frac{e^{-2 m_{e}\left|\mathbf{x}_{3}-\mathbf{x}_{4}\right|}}{\left|\mathbf{x}_{3}-\mathbf{x}_{4}\right|} \times
$$




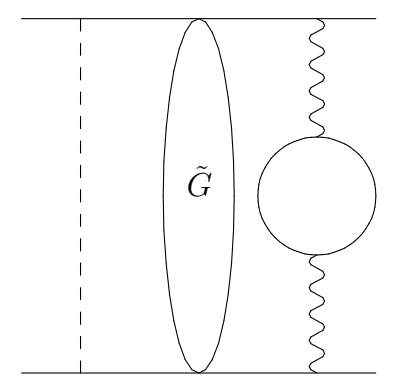

FIG. 4: Vacuum polarization effects in second order perturbation theory. The dashed line represents first part of the potential $\Delta H(3)$. The wave line represents the hyperfine part of the Breit potential.

$$
\times \sum_{n^{\prime} \neq 0} \frac{\psi_{e, n^{\prime}}\left(\mathbf{x}_{3}\right) \psi_{e, n^{\prime}}^{*}\left(\mathbf{x}_{1}\right)}{E_{e}-E_{e n^{\prime}}} \Delta V_{\mu}\left(\mathbf{x}_{1}\right) \psi_{e}\left(\mathbf{x}_{1}\right)
$$

where the matrix elements entering in (66) are defined by (9) and (57). Integrating over all coordinates in (66) and summing it with (65) we obtain the following convergent integral in $\xi$ in the leading order with respect to the ratio $\left(M_{e} / M_{\mu}\right)$ :

$$
\begin{gathered}
\Delta \nu_{V P, 11,2 S}^{h f s,(2)}+\Delta \nu_{V P, 12,2 S}^{h f s,(2)}=\nu_{F} \frac{\alpha\left(1+\kappa_{\mu}\right) M_{e}}{3 \pi M_{\mu}} \int_{1}^{\infty} \frac{\rho(\xi) d \xi}{\left(a_{2}+1\right)^{8}}\left(51 a_{2}^{6}+408 a_{2}^{5}+\right. \\
\left.+1177 a_{2}^{4}+1872 a_{2}^{3}+2029 a_{2}^{2}+1464 a_{2}+631\right)=0.004 \text { MHz }
\end{gathered}
$$

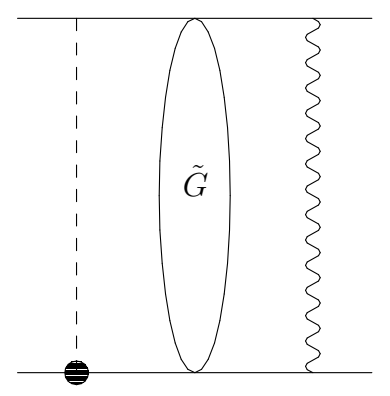

FIG. 5: Nuclear structure effects in second order perturbation theory. The bold point represents the nuclear vertex operator. The wave line represents the hyperfine part of the Breit potential.

Next correction arises from the muon in $2 P$ intermediate state. Making the same division of total contribution into two parts as in (67) we present here final result:

$$
\begin{gathered}
\Delta \nu_{V P, 11,2 P}^{h f s,(2)}+\Delta \nu_{V P, 12,2 P}^{h f s,(2)}=-\nu_{F} \frac{3 \alpha\left(1+\kappa_{\mu}\right) M_{e}}{256 \pi M_{\mu}} \int_{1}^{\infty} \frac{\rho(\xi) d \xi}{\left(a_{2}+1\right)^{8}}\left[5 a_{2}^{6}+40 a_{2}^{5}+139 a_{2}^{4}+\right. \\
\left.+272 a_{2}^{3}+323 a_{2}^{2}+232 a_{2}+93\right]=-0.005 \text { MHz. }
\end{gathered}
$$

Let us consider now the terms with $n \neq 2 S, 2 P$ :

$$
\Delta \nu_{V P, \mu-e, n \neq 2 S, 2 P}^{h f s,(2)}=-\frac{16 \alpha^{3}\left(1+\kappa_{\mu}\right) M_{e}}{18 \pi^{2} m_{e} m_{\mu}} \int_{1}^{\infty} \rho(\xi) d \xi \int d \mathbf{x}_{1} \int d \mathbf{x}_{2} \int d \mathbf{x}_{3} \int d \mathbf{x}_{4} \psi_{\mu}^{*}\left(\mathbf{x}_{4}\right) \psi_{e}^{*}\left(\mathbf{x}_{3}\right) \times
$$




$$
\left[\pi \delta\left(\mathbf{x}_{3}-\mathbf{x}_{4}\right)-\frac{m_{e}^{2} \xi^{2}}{\left|\mathbf{x}_{3}-\mathbf{x}_{4}\right|} e^{-2 m_{e} \xi\left|\mathbf{x}_{3}-\mathbf{x}_{4}\right|}\right] \sum_{n \neq 2 S, 2 P} \psi_{n, \mu}\left(\mathbf{x}_{4}\right) \psi_{\mu, n}^{*}\left(\mathbf{x}_{2}\right) \frac{e^{-b\left|\mathbf{x}_{3}-\mathbf{x}_{1}\right|}}{\left|\mathbf{x}_{3}-\mathbf{x}_{1}\right|\left|\mathbf{x}_{2}-\mathbf{x}_{1}\right|} \psi_{\mu}\left(\mathbf{x}_{2}\right) \psi_{e}\left(\mathbf{x}_{1}\right)
$$

where free Green's function of the electron (15) is used. Changing $\psi_{e}\left(\mathbf{x}_{1}\right) \rightarrow \psi_{e}(0)$ and using first nonzero term in the expansion (16) we present the contribution of the $\delta$-function from (69) in the form:

$$
\Delta \nu_{V P, 11, n \neq 2 S, 2 P}^{h f s,(2)}=-\nu_{F} \frac{\alpha\left(1+\kappa_{\mu}\right) M_{e}}{3 \pi M_{\mu}} \int_{1}^{\infty} \rho(\xi) d \xi \frac{665}{128}
$$

As expected the integral (70) in $\xi$ is divergent. We should consider together with it another term from (69):

$$
\begin{gathered}
\Delta \nu_{V P, 12, n q \zeta, 2 P}^{h f s,(2)}=-\nu_{F} \frac{2 \alpha^{2}\left(1+\kappa_{\mu}\right) M_{e} m_{e}^{2}}{3 \pi^{2}} \int_{1}^{\infty} \rho(\xi) d \xi \int d \mathbf{x}_{2} \int d \mathbf{x}_{3} \int d \mathbf{x}_{4} \frac{e^{-b\left|\mathbf{x}_{3}-\mathbf{x}_{4}\right|}}{\left|\mathbf{x}_{3}-\mathbf{x}_{4}\right|} \psi_{\mu}\left(\mathbf{x}_{4}\right) \times \\
{\left[\delta\left(\mathbf{x}_{2}-\mathbf{x}_{4}\right)-\psi_{\mu}\left(\mathbf{x}_{2}\right) \psi_{\mu}^{*}\left(\mathbf{x}_{4}\right)-\sum_{m=-1}^{1} \psi_{\mu, 2 P, m}\left(\mathbf{x}_{2}\right) \psi_{\mu, 2 P, m}^{*}\left(\mathbf{x}_{4}\right)\right]\left|\mathbf{x}_{3}-\mathbf{x}_{2}\right| \psi_{\mu}\left(\mathbf{x}_{2}\right) .}
\end{gathered}
$$

All three terms in square brackets give the contributions in (71). They can be evaluated in the same way as in previous sections. The sum of expressions (70) and (71) is determined by finite integral which is calculated numerically:

$$
\begin{aligned}
& \Delta \nu_{V P, 11, n \neq 2 S, 2 P}^{h f s,(2)}+\Delta \nu_{V P, 12, n \Varangle S, 2 P}^{h f s,(2)}=\nu_{F} \frac{\alpha\left(1+\kappa_{\mu}\right) M_{e}}{3 \pi M_{\mu}} \int_{1}^{\infty} \frac{\rho(\xi) d \xi}{128 a_{2}\left(a_{2}+1\right)^{9}}\left(-5 a_{2}^{9}+16 a_{2}^{8}+(72)\right. \\
& \left.+403 a_{2}^{7}+2075 a_{2}^{6}+4596 a_{2}^{5}+7310 a_{2}^{4}+6559 a_{2}^{3}+4511 a_{2}^{2}+1639 a_{2}+256\right)=0.012 \mathrm{MHz} .
\end{aligned}
$$

All vacuum polarization contributions to the hyperfine splitting are presented in Table I. In their calculation we use systematically the expansion in $M_{e} / M_{\mu}$ retaining first order terms. So, all corrections evaluated in this section are of second order in two small parameters $\alpha$ and $M_{e} / M_{\mu}$.

\section{NUCLEAR STRUCTURE CORRECTIONS}

In the leading order in $\alpha$ the nuclear structure corrections to hyperfine splitting are determined by the charge radius of $\alpha$-particle $r_{\alpha}$. Considering the interaction between the muon and the nucleus we can present the nuclear structure correction in the interaction operator in the form [6]:

$$
\Delta V_{s t r, \mu-\alpha}\left(\mathbf{r}_{\mu}\right)=\frac{2}{3} \pi Z \alpha<r_{\alpha}^{2}>\delta\left(\mathbf{r}_{\mu}\right)
$$

where the subscript str designates the structure correction. The contribution of the operator $\Delta V_{s t r, \mu-\alpha}$ to the hyperfine splitting appears in second order perturbation theory (see the diagram in Fig $[5$. We can write it in the integral form:

$$
\Delta \nu_{s t r, \mu-\alpha}^{h f s}=\frac{64 \pi^{2} \alpha^{2}\left(1+\kappa_{\mu}\right) r_{\alpha}^{2}}{9 m_{e} m_{\mu}} \frac{W_{\mu}^{3 / 2}}{2 \sqrt{2 \pi}} \int d \mathbf{x}_{3} \psi_{\mu}^{*}\left(\mathbf{x}_{3}\right)\left|\psi_{e}\left(\mathbf{x}_{3}\right)\right|^{2} G_{\mu}\left(\mathbf{x}_{3}, 0, E_{\mu}\right) .
$$


TABLE I: Hyperfine singlet-triplet splitting of the excited state in muonic helium atom.

\begin{tabular}{|c|c|c|}
\hline Contribution to the HFS & $\Delta \nu^{h f s}, \mathrm{MHz}$ & Reference \\
\hline The Fermi splitting & 4516.915 & $(5)$ \\
\hline $\begin{array}{l}\text { The muon anomalous magnetic } \\
\text { moment correction } a_{\mu} \nu_{F}\end{array}$ & 5.266 & $(5)$ \\
\hline $\begin{array}{l}\text { Recoil corrections in } M_{e} / M_{\mu} \\
\text { in first order } \mathrm{PT}\end{array}$ & -132.608 & $(5)$ \\
\hline $\begin{array}{c}\text { Recoil correction in } M_{e} / M_{\mu} \text { in second } \\
\text { order PT, muon } 2 S \text { state }\end{array}$ & 61.127 & $(10)$ \\
\hline $\begin{array}{c}\text { Recoil correction in } M_{e} / M_{\mu} \text { in second } \\
\text { order PT, muon } 2 P \text { state }\end{array}$ & -70.919 & $(13)$ \\
\hline $\begin{array}{l}\text { Recoil correction in } M_{e} / M_{\mu} \text { in second } \\
\text { order } \mathrm{PT} \text {, muon excited states }\end{array}$ & -109.978 & $(18),(21)$ \\
\hline $\begin{array}{c}\text { Recoil correction of order } \frac{M_{e}}{m_{\alpha}} \sqrt{\frac{M_{e}}{M_{\mu}}} \alpha^{4} \text { in second } \\
\text { order PT, muon excited states }\end{array}$ & 0.189 & $(27)$ \\
\hline $\begin{array}{l}\text { Recoil correction of order } \frac{M_{e}^{2}}{m_{\alpha} M_{\mu}} \alpha^{4} \text { in second } \\
\text { order PT, muon excited states }\end{array}$ & 0.074 & $(29)$ \\
\hline $\begin{array}{l}\text { Recoil correction of order } \\
\alpha^{5}\left(m_{e} / m_{\mu}\right) \ln \left(m_{e} / m_{\mu}\right)\end{array}$ & 0.812 & $(31),[15]$ \\
\hline $\begin{array}{c}\text { Recoil correction } \frac{69 m_{e}^{2}}{2 m_{\mu}^{2}} \ln \frac{m_{\mu}}{m_{e}} \nu_{F} \\
\text { in third order PT }\end{array}$ & 19.434 & {$[14]$} \\
\hline $\begin{array}{c}\text { Electron vertex correction of order } \alpha^{5} \\
\text { in first order PT }\end{array}$ & 5.078 & $(33)$ \\
\hline $\begin{array}{c}\text { Electron vertex correction of order } \alpha^{5} \\
\text { in second order PT }\end{array}$ & 0.750 & $(36),(40),(43)$ \\
\hline $\begin{array}{l}\text { One-loop VP contribution in } 1 \gamma \\
(e \mu) \text { interaction of order } \alpha^{5}\end{array}$ & 0.012 & $(49)$ \\
\hline $\begin{array}{l}\text { One-loop VP contribution in the } \\
\text { electron-nucleus interaction in the } \\
\text { second order PT of order } \alpha^{5}\end{array}$ & 0.028 & $(52)$ \\
\hline $\begin{array}{l}\text { One-loop VP contribution in the } \\
\text { muon-nucleus interaction in the } \\
\text { second order PT of order } \alpha^{5}\end{array}$ & 0.099 & $(55)$ \\
\hline $\begin{array}{l}\text { One-loop VP contribution in the } \\
\text { electron-muon interaction in the } \\
\text { second order PT of order } \alpha^{5}\end{array}$ & 0.059 & $\begin{array}{c}(58),(61),(64),(67), \\
(68),(72)\end{array}$ \\
\hline $\begin{array}{c}\text { Nuclear structure correction of order } \\
\alpha^{6} \text { in second order PT }\end{array}$ & -0.015 & $(75),(76)$ \\
\hline Relativistic correction of order $\alpha^{6}$ & -0.050 & {$[15]$} \\
\hline Electron vertex correction of order $\alpha^{6}$ & -0.615 & {$[6,28-30]$} \\
\hline Summary contribution & 4295.658 & \\
\hline
\end{tabular}


Using the muon Green's function with one zero argument we make analytical integration in (174) and present final result in the form:

$$
\Delta \nu_{s t r, \mu-\alpha}^{h f s}=-\nu_{F} \frac{8 \alpha^{2} M_{\mu}^{2} r_{\alpha}^{2}\left(1+\kappa_{\mu}\right)}{3}\left(6 \frac{M_{e}}{M_{\mu}}-41 \frac{M_{e}^{2}}{M_{\mu}^{2}}+\ldots\right)=-0.014 \mathrm{MHz} .
$$

Numerical value is obtained by means of the charge radius of the $\alpha$-particle $r_{\alpha}=1.676 \mathrm{fm}$. The same approach can be used in the study of the electron-nucleus interaction. The electron feels as well the distribution of the electric charge of $\alpha$ particle. The corresponding contribution of the nuclear structure effect to the hyperfine splitting is the following:

$$
\Delta \nu_{s t r, e-\alpha}^{h f s}=-\nu_{F} \frac{4 \alpha^{2} M_{\mu}^{2} r_{\alpha}^{2}}{3}\left[\frac{1}{2} \frac{M_{e}}{M_{\mu}}-\frac{M_{e}^{2}}{M_{\mu}^{2}}\left(\frac{3}{2}+\ln \frac{M_{e}}{M_{\mu}}\right)+\ldots\right]=-0.001 \mathrm{MHz} .
$$

We have included in Table I the total nuclear structure contribution which is equal to the sum of numerical values (75) and (76) .

\section{CONCLUSION}

In this work we have performed the analytical and numerical calculation of some basic contributions to the hyperfine splitting of the excited state $1 s_{1 / 2}^{(e)} 2 s_{1 / 2}^{(\mu)}$ in muonic helium atom connected with the recoil effects, electron vertex corrections, vacuum polarization and nuclear structure effects. We used the perturbation theory for this task as it was formulated previously in [8]. As was shown by Lakdawala and Mohr that the ground state hyperfine splitting in muonic helium atom can be expressed analytically by a series in the ratio $M_{e} / M_{\mu}$. We extend their method to the case of the excited state $1 s_{1 / 2}^{(e)} 2 s_{1 / 2}^{(\mu)}$ accounting for not only purely recoil corrections but also other vacuum effects controlled by usual small parameter $\alpha$ in QED. Our results are presented in Table $\mathrm{I}$ in which we give also references to the calculations of some other corrections which are not considered here. We have included in the total value of the hyperfine splitting the recoil logarithmic correction obtained in [14] in third order PT, the relativistic correction obtained in [15], the electron vertex correction of order $O\left(\alpha^{2}\right)$ known from the theory of hydrogenic atoms [6, 28 -30]. Our numerical result $4295.66 \mathrm{MHz}$ is, in whole, in the agreement with the earlier performed calculations by the perturbation theory and variational method: $4291.50 \mathrm{MHz}$ [14], 4287.01 $\pm 0.10 \mathrm{MHz}$ [15]. Our analytical expressions of different recoil corrections are in agreement with the calculation in [14]. Comparing our results (10), (13), (18), (21), (29) with [14] we can find their coincidence in the leading order in $m_{e} / m_{\mu}, m_{e} / m_{\alpha}$ and logarithmic terms $\frac{m_{e}^{2}}{m_{\mu}^{2}} \ln \frac{m_{\mu}}{m_{e}}$. We should take into account that the used definition of reduced masses $M_{e}$ and $M_{\mu}$ of the particles is different from $m_{e}^{*}$ and $m_{\mu}^{*}$ in [14]. The correspondence between our results and basic expression (62) from [14] can be revealed if we use the relation: $M_{e} / M_{\mu}=m_{e}^{*} / m_{\mu}+$ $m_{e}^{*} / m_{\alpha}$. It is interesting to note that the recoil correction of order $m_{e} / m_{\alpha}$ appears in our kinematics from the definitions of reduced masses $M_{e}$ and $M_{\mu}$. The recoil Hamiltonian $\Delta H_{\text {rec }}$ gives the contribution in second order perturbation theory of order $M_{e}^{2} / m_{\alpha} M_{\mu}$ (29). Numerical differences of our results from [14] are determined by different choice of threeparticle kinematics, higher order corrections $M_{e}^{2} / M_{\mu}^{2}$ which we account for in intermediate expressions and calculated vacuum polarization effects of order $\alpha M_{e} / M_{\mu}$. So, for example, 
numerical value of the recoil correction of the leading order $\left(-6 \frac{M_{e}}{M_{\mu}}\right) \nu_{F}$ in (I5) is equal to $-134.769 \mathrm{MHz}$. This number differs by more than $2 \mathrm{MHz}$ from the value $-132.608 \mathrm{MHz}$ presented in Table I because we preserve corrections of higher order presenting (5) in the form $\nu_{F}\left(1+\kappa_{\mu}\right)\left(1-6 M_{e} / M_{\mu}+21 M_{e}^{2} / M_{\mu}^{2}-55 M_{e}^{3} / M_{\mu}^{3}\right)$. Another important source of the difference is specified by more accurate calculation of the electron vertex correction in section III related with the Pauli form factor. We use exact expression for it making numerical integration in corresponding amplitudes. Finally, we use in hyperfine splitting part of the Hamiltonian the factor $\left(1+\kappa_{\mu}\right)$ related with the muon anomalous magnetic moment what also gives definite modifications of numerical results. Our summary value of the vacuum polarization effects $0.198 \mathrm{MHz}$ agrees with the result $0.229 \mathrm{MHz}$ obtained by variational method calculation in [15].

Effects of the nuclear structure give negligibly small contribution to hyperfine splitting. In muonic helium atom the muon strongly interacts with $\alpha$-particle at distances of order $r \sim 1 / M_{\mu} \alpha$. It was discovered recently in the muonic hydrogen Lamb shift experiment that the experimental value differs from theoretical one on $0.3 \mathrm{meV}$ with CODATA value of the proton charge radius [31]. This disagreement between the proton charge radius extracted from Lamb shift measurements of muonic and electronic hydrogen can be considered as the appearance of new muon physics [32]. The plans to measure the $(2 S-2 P)$ transition frequencies in muonic helium ions $\left(\mu_{2}^{4} \mathrm{He}\right)^{+}$and $\left(\mu_{2}^{3} \mathrm{He}\right)^{+}$with an accuracy of $50 \mathrm{ppm}$ can help to solve the proton radius puzzle [33]. In the case of muonium the most precise comparison between theory and experiment is possible [6, 34]. It will be interesting to use muonic helium atom for the comparison of theoretical value of hyperfine splitting $4295.66 \mathrm{MHz}$ with experimental data for the revealing of new interactions in muonic sector. Main theoretical error in our calculation is connected with uncalculated in full recoil contribution

of second order $\nu_{F} \frac{M_{e}^{2}}{M_{\mu}^{2}} \approx 0.11 \mathrm{MHz}$. In addition, the electron vertex corrections in twophoton processes which are estimated in this work very approximately also give essential error $\nu_{F} \alpha^{2} \approx 0.25 \mathrm{MHz}$. Thereby, the total theoretical error does not exceed $\pm 0.40 \mathrm{MHz}$, but large values of coefficients can essentially change preliminary estimate. Further improvements of the theoretical result require more careful construction of the three-particle interaction operator connected with the multiphoton exchanges.

\section{Acknowledgments}

This work is supported by the Russian Foundation for Basic Research (grant No.11-0200019) and the Ministry of Education and Science of Russian Federation (government order for Samara State U. No. 2.870.2011).

[1] H. Orth et al, Phys. Rev. Lett. 45, 1483 (1980).

[2] C.J. Gardner et al., Phys. Rev. Lett. 48, 1168 (1982).

[3] H.A. Bete and E.E. Salpeter, Quantum Mechanics of One- and Two-Electron Atoms (Springer, Berlin, 1957).

[4] J.R. Sapirstein and D.R. Yennie, in Quantum Electrodynamics, edited by T. Kinoshita (World Scientific, Singapore, 1990), p.560

[5] K. Pachucki, Ann. Phys. 226, 1 (1993). 
[6] M.I. Eides, H. Grotch, and V.A. Shelyuto, Phys. Rep. 342, 62 (2001).

[7] S.G. Karshenboim, Phys. Rep. 422, 1 (2005).

[8] S.D. Lakdawala and P.J. Mohr, Phys. Rev. A 22, 1572 (1980); Phys. Rev. A 29, 1047 (1984); Phys. Rev. A 24, 2224 (1981).

[9] K.-N. Huang and V.W. Hughes, Phys. Rev. A 20, 706 (1979); Phys. Rev. A 26, 2330 (1982).

[10] A.M. Frolov, Phys. Rev. A 61, 022509 (2000).

[11] E. Borie, Z. Phys. A 291, 107 (1979).

[12] K. Pachucki, Phys. Rev. A 63, 032508 (2001).

[13] R.L. Drachman, Phys. Rev. A 22, 1755 (1980); J. Phys. B 16, L749 (1983).

[14] V.L. Yakhontov and M.Ya. Amusia, J. Phys. B 27, 3743 (1994); M.Ya. Amusia, M.Ju. Kuchiev, and V.L. Yakhontov, J. Phys. B 16, L71 (1983).

[15] M.-K. Chen, J. Phys. B 26, 2263 (1993); Phys. Rev. A 45, 1479 (1992).

[16] S. Barcza, Z. Phys. D 24, 223 (1992).

[17] P.J. Mohr, B.N. Taylor, and D.B. Newell, arXiv:1203.5425 [physics.atom-ph]

[18] A.P. Martynenko, Phys. Rev. A 71, 022506 (2005); JETP 101, 1021 (2005).

[19] A.P. Martynenko, Phys. Rev. A 76, 012505 (2007); JETP 133, 742 (2008).

[20] E.N. Elekina and Martynenko, Phys. Atom. Nucl. 73, 1828 (2010); A.P. Martynenko and E.N. Elekina, Phys. Atom. Nucl. 73, 2074 (2010); A.A. Krutov and A.P. Martynenko, Phys. Rev. A 84, 052514 (2011).

[21] U.D. Jentschura, Ann. Phys. 326, 500 (2011); Ann. Phys. 326, 516 (2011).

[22] A.A. Krutov and A.P. Martynenko, Phys. Rev. A 78, 032513 (2008); Eur. Phys. J. D 62, 163 (2011).

[23] H.F. Hameka, Jour. Chem. Phys. 47, 2728 (1967).

[24] B.R. Johnson and J.O. Hirschfelder, J. Math. Phys. 20, 2484 (1979).

[25] V.A. Fok, Principles of Quantum Mechanics ( Nauka, Moscow, 1976).

[26] R. Arnowitt, Phys. Rev. 92, 1002 (1953).

[27] V.B. Berestetskii, E.M. Lifshits, and L.P. Pitaevskii, Quantum Electrodynamics (Nauka, Moscow, 1980).

[28] S.J. Brodsky and G.W. Erickson, Phys. Rev. 148, 26 (1966).

[29] N. Kroll and F. Pollack, Phys. Rev. 84, 594 (1951).

[30] R. Karplus, A. Klein, and J. Schwinger, Phys. Rev. 84, 597 (1951).

[31] R. Pohl, A. Antognini, F. Nez et al., Nature 466, 213 (2010).

[32] C.E. Carlson and B.C. Rislow, arXiv:1206.3587 [hep-ph].

[33] A. Antognini, F. Biraben, J.M.R. Cardoso et al., Can. J. Phys. 89, 47 (2011).

[34] M.I. Eides and V.A. Shelyuto, Phys. Rev. A 86, 024501 (2012). 